\title{
A System For Obtaining High Resolution Macroscopic Surface Charge Density Distributions On Contoured Axi-Symmetric Insulator Specimens
}

\author{
D C Faircloth and N L Allen \\ Department of Electrical and Electronic Engineering, UMIST, UK
}

\begin{abstract}
This paper describes the method of operation of a system for measuring high-resolution surface charge density maps on practical insulator specimens using an electrostatic probe. Apparatus is described that can scan the electrostatic probe over the surface of the insulator and record the probe signal. The process of obtaining a charge density map from the probe signal measurements is explained. An example charge distribution is shown.
\end{abstract}

\section{Introduction}

Atmospheric air provides the basic insulation for many practical high voltage installations such as transmission lines, switchgear etc. However, the high voltage conductor in any such system must be mechanically supported, and the only practical solution is to use solid insulation. This introduces an insulating surface between the high voltage conductor and ground. An insulator's surface is intrinsically the weakest part of the solid-gas insulation system. Thus, physical knowledge of the insulating properties of solid insulation surfaces is very important. A major factor which influences surface discharges is the charge deposited on the surface of the solid insulation. This has led to the development of the surface charge scanning apparatus described in this paper. The apparatus is capable of measuring high-resolution charge density maps on all or part of almost any contoured axi-symmetric insulator specimen.

\section{Surface Charge Density Measurement}

\subsection{Electrostatic Probe}

The electrostatic probe principle [1] is used, where charge on the surface induces a voltage on the centre conductor of a coaxial probe positioned above the surface. The outer conductor is grounded and the voltage induced on the centre conductor is measured via a very high input impedance $\left(>10^{15} \Omega\right)$ op-amp. The outer diameter of the probe is $2.9 \mathrm{~mm}$ and the diameter of centre conductor is $0.6 \mathrm{~mm}$. A multi-point measuring technique is employed, where the probe voltage is recorded at many points above the insulator surface. 


\subsection{Calibration Problem}

The probe voltage measurements must be converted into surface charge density measurements. Early investigators [1] used a simple capacitive method in which each value was linearly related to a charge density. This technique yields limited accuracy and makes no account for the probe's response to neighbouring charges on the surface. In recent years techniques have evolved to model the probe response to distant charges [2]. The technique used here is an adaptation of Pedersen's $\lambda$-function [3]. He related the Poisionian charge $(\mathrm{q})$ induced on the probe to the surface charge density $(\sigma)$ on a surface element: $q=\lambda \sigma$.

The technique employed here relates contribution to the total probe voltage (v) directly to the surface charge density $(\sigma)$ on a surface element: $v=\phi \sigma$, where $\phi$ is the constant of proportionality measured in $\mathrm{Cm}^{-2} \mathrm{~V}^{-1}$. Each element has a different associated $\phi$-value depending on its distance from the probe. The total probe voltage (V) is the sum of the contributions from all the elements of surface charge: $\mathrm{V}=\Sigma \mathrm{v}=\Sigma \phi \sigma$. For the probe in one particular position the $\phi$-values for all the elements on the surface make up the probe response function. The probe has a different response function for each voltage measurement position.

\subsection{The Ф-Matrix Technique}

The surface area is divided into elements as shown in figure 1. The elements do not have to be square and there does not have to be an equal number of horizontal and vertical divisions.

The probe voltage in position $(i, j)$ is given by:

$$
v_{i j}=\sum_{n_{y}}^{y=1}\left[\sum_{n_{x}}^{x=1} \phi_{i j}(x y) \sigma_{x y}\right]
$$

where, $\phi_{i j}(x y)$ is the value of the probe's response function to charge at position $(\mathrm{x}, \mathrm{y})$ for the probe at position $(\mathrm{i}, \mathrm{j})$ and, $\sigma_{x y}$ is the surface charge density on the surface element at position $(\mathrm{x}, \mathrm{y})$. This is a first order function of the $n_{x} n_{y}$ surface charge densities.

There are $n_{x} n_{y}$ probe voltage measurements in total and each of these voltages is a function of $n_{x} n_{y}$ surface charge densities. The problem is reduced to the solution of $n_{x} n_{y}$ simultaneous equations, which is solved using the matrix inversion technique.

The measured probe voltages and the unknown charge densities are grouped to two vectors $\overline{\boldsymbol{V}}$ and $\overline{\boldsymbol{\sigma}}$. They are related by the matrix equation: $\quad \bar{V}=\overline{\boldsymbol{\sigma}} \Phi$

where, $\Phi$ is a matrix containing all the $\phi$-function values that are coefficients of the simultaneous equations. Hence the unknown charge density's can be found from: $\bar{\sigma}=\bar{V} \Phi^{-1}$

Surface is divided into $\mathrm{n}_{\mathrm{x}} \times \mathrm{n}_{\mathrm{y}}$ elements numbered from the top left corner

Specific surface elements can be identified using the variables $(x, y)$

The element which the probe is directly above is identified using the variables $(i, j)$

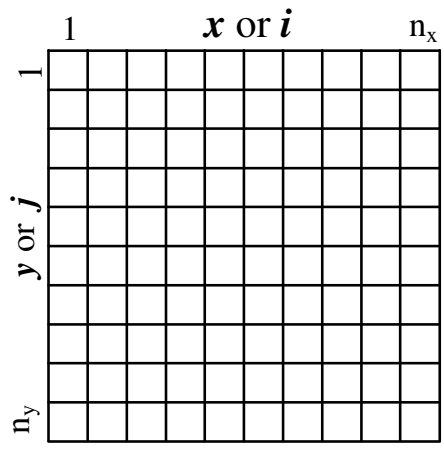

Figure 1: The division of the surface 

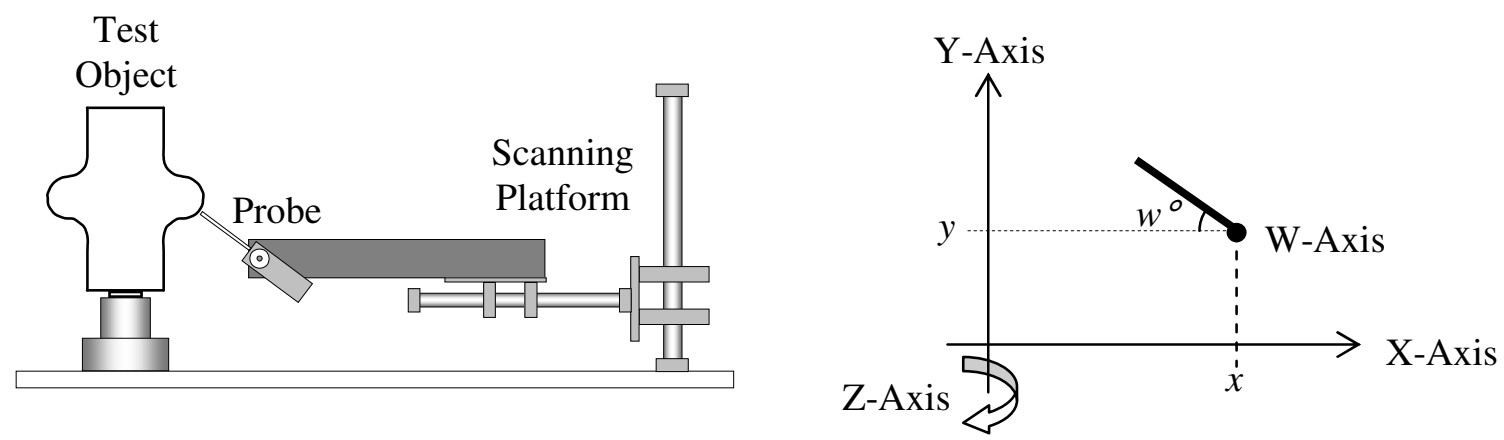

Figure 2: The scanning apparatus and the four axes that define the position of the probe above the surface.

\section{The Scanning Apparatus}

The probe is positioned using 4-stepper motors, as shown in figure2. The scanning platform was designed and constructed at UMIST. A computer controls the whole scanning system using specially written software. The contour of the test object can be entered into the computer manually or the system can measure the shape of the test object automatically using a spring-loaded sensor. The computer then calculates the probe positions to scan the surface. The probe must be kept perpendicular to, and a constant distance from, the surface. The surface is divided up into measurement points at which the probe voltage is recorded.

The surface is scanned in layers by rotating the test object. After each rotation the probe moves to the start position of the next layer until the whole surface has been scanned.

As the probe moves over the surface the probe voltage is measured at each point and stored in a file on the computer. All the settings for the scanning system are also stored in the same file; this allows the user to recall measurement parameters at a later date. The software has the facility to graphically display the scanned in voltage measurements and monitor each layer as it is scanned in. The scanning system has been developed as a versatile, easy to use piece of equipment with many useful facilities to help the user.

\section{Implementing The Calibration Technique}

The probe's response functions are found using a 3-D electrostatic field solver, key values are found, then a computer generates the $\Phi$-matrix by quadraticaly interpolating any missing $\phi$-values.

The matrix is inverted in Matlab running under UNIX on a Fujitsu AP3000. The solution of the charge distributions is automated using script files; files generated by the scanning system are read in, the required $\Phi$-matrix is automatically generated and the charge distributions are solved and saved, all without any user intervention.

The solution time increases with the resolution of the surface. As the number of surface divisions increases so does the size of the matrix required to solve the charge distribution. The amount of computer memory required increases as $\mathrm{n}^{4}$ where $\mathrm{n}$ is the 1-dimensional division of the surface (i.e. a surface is divided into $\mathrm{n} \times \mathrm{n}$ elements). For example a surface scanned to a $100 \times 100$ resolution requires a $10,000 \times 10,000 \Phi$-matrix which needs $800 \mathrm{MB}$ of RAM and takes just under 24 hours to solve. The amount of RAM required increases very quickly with resolution; for a $150 \times 150$ surface division 4GB of RAM is required. 


\section{Example Surface Scans}

To demonstrate the resolution of the system, a scan is made of the corona produced by a single $-10 \mathrm{kV} 1.2 / 50 \mu$ s impulse applied to a point electrode $1 \mathrm{~mm}$ above the surface of a cylindrical PTFE insulator specimen. The effect of calibration using the $\Phi$-Matrix technique is clearly visible by the differences between figures 3 and 4 . After the surface was scanned a dust figure was obtained (figure 6) using black photocopier toner which adheres to positive charge. The dust figure compares favourably with the charge density contour map (figure 5).

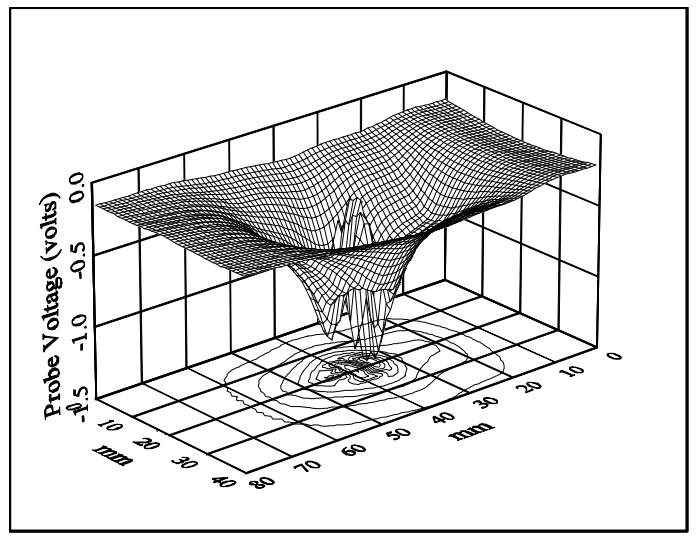

Figure 3: The measured probe voltage

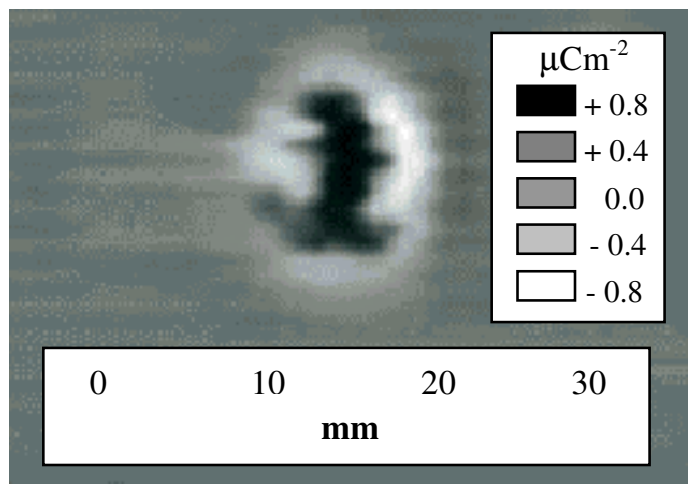

Figure 5: Surface charge contour map

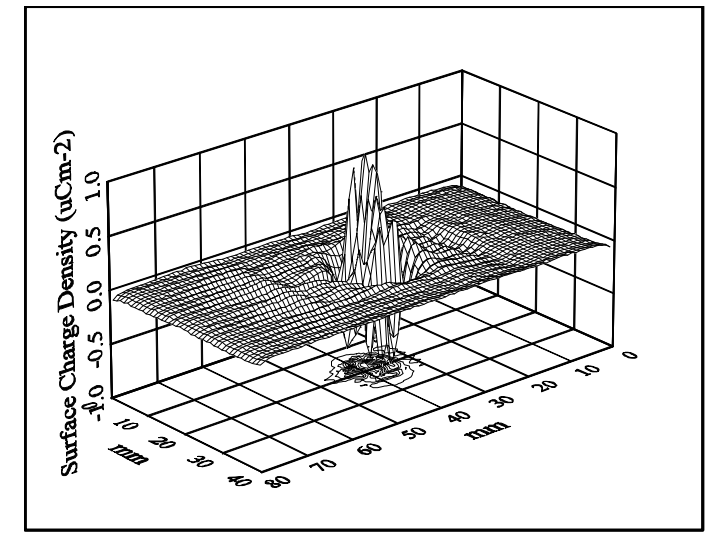

Figure 4: The calibrated surface charge density

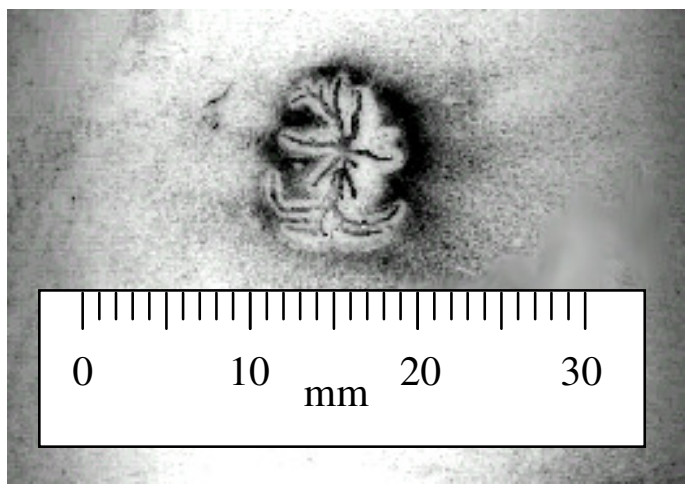

Figure 6: Dust figure obtained after surface had been scanned.

\section{References}

[1] D.K. DAVIES, "The Examination of the Electrical Properties of Insulators by Surface Charge Measurement", Journal of Scientific Instrumentation, Vol. 44, pp. 521-524, 1967.

[2] T.O. Rerup, et al, "Using the $\lambda$ Function to Evaluate Probe Measurements of Charged Dielectric

Surfaces", IEEE Trans. Dielectrics EI, Vol. 3, pp. 770-777, 1996.

[3] A. Pedersen, 'On the Electrostatics of Probe Measurements of Surface Charge Densities', Gaseous Dielectrics V, Pergamon Press, pp. 235-240, 1987. 